\title{
Production monitoring system for understanding product robustness
}

\author{
Boorla, S.M. ${ }^{\mathrm{a},{ }^{*}}$, Howard, T.J. ${ }^{\mathrm{a}}$ \\ ${ }^{a}$ Technical University of Denmark, Department of Mechanical Engineering, Denmark
}

\begin{abstract}
A B S T R A C T
In the current quality paradigm, the performance of a product is kept within specification by ensuring that its parts are within specification. Product performance is then validated after final assembly. However, this does not control how robust the product performance is, i.e. how much it will vary between the specification limits. In this paper, a model for predicting product performance is proposed, taking into account design, assembly and process parameters live from production. This empowers production to maintain final product performance, instead of part quality. The PRECI-IN case study is used to demonstrate how the monitoring system can be used to efficiently guide corrective action to improve product performance. It is claimed that the monitoring system can be used to dramatically cut the time taken to identify, plan and execute corrective action related to typical quality issues. To substantiate this claim, two further cases comparable to PRECI-IN, in terms of complexity, material and manufacturing process, were taken from different industries. The interviews with quality experts revealed that the typical time taken for corrective action for both cases was accounted to be seven days. Using the monitoring system for the PRECI-IN case, similar corrective action would have been achieved almost immediately.
\end{abstract}

\section{ARTICLE INFO}

Keywords:

Product robustness

Performance variation

Robustness monitoring system

Performance consistency

Unit to unit robustness

*Corresponding author:

srimbo@mek.dtu.dk

(Boorla, S.M.)

Article history:

Received 27 May 2016

Revised 5 August 2016

Accepted 17 August 2016

(C) 2016 PEI, University of Maribor. All rights reserved.

\section{References}

[1] Helten, K., Hellenbrand, D., Lindemann, U. (2009). Product robustness as a basis for the improvement of production planning processes-key factors in early design phases, In: DS 58-7: Proceedings of ICED 09, the 17th International Conference on Engineering Design, Palo Alto, CA, USA, 197-206.

[2] Howard, T.J., Ebro, M., Eifler, T., Göhler, S.M., Pedersen, S.N., Christiansen, A., Rafn, A. (2014). The variation management framework (VMF) for robust design, In: $1^{\text {st }}$ International Symposium on Robust Design, Technical University of Denmark, Copenhagen, Denmark, 171-175, doi: 10.4122/dtu:2104.

[3] Saha, A., Ray, T. (2011). Practical robust design optimization using evolutionary algorithms, Journal of Mechanical Design, Vol. 133, No. 10, 101012-101012-19, doi: 10.1115/1.4004807.

[4] Ebro, M., Howard, T.J. (2016). Robust design principles for reducing variation in functional performance, Journal of Engineering Design, Vol. 27, No. 1-3, 75-92, doi: 10.1080/09544828.2015.1103844.

[5] Ebro, M., Howard, T.J., Rasmussen, J.J. (2012). The foundation for robust design: Enabling robustness through kinematic design and design clarity, In: DS 70: Proceedings of Design 2012, the $12^{\text {th }}$ International Design Conference, Dubrovnik, Croatia, 817-826.

[6] Göhler, S.M., Howard, T.J. (2014). Framework for the application of robust design methods and tools, In: Proceedings of the First International Symposium of Robust Design 2014, Technical University of Denmark, Copenhagen, Denmark, 123-133, doi: 10.4122/dtu:2099.

[7] Montgomery, D.C. (2009), Statistical quality control, Wiley, New York, USA.

[8] Xie, M., Lu, X.S., Goh, T.N., Chan, L.Y. (1999). A quality monitoring and decision-making scheme for automated production processes, International Journal of Quality \& Reliability Management, Vol. 16, No. 2, 148-157, doi: 10.1108/02656719910218238. 
[9] El-Midany, T.T., El-Baz, M.A., Abdelwahed, M.S. (2013). Improve characteristics of manufactured products using artificial neural network performance prediction model, International Journal of Recent Advances in Mechanical Engineering, Vol. 2, No. 4, 23-34.

[10] Zhang, C., Liu, X., Shi, J., Zhu, J. (2006). Neural soft-sensor of product quality prediction, In: $6^{\text {th }}$ World Congress on Intelligent Control and Automation, Dalian, China, 4881-4885, doi: 10.1109/WCICA.2006.1713312.

[11] Frey, D.D., Li, X. (2008). Using hierarchical probability models to evaluate robust parameter design methods, Journal of Quality Technology, Vol. 40, No. 1, 59-77.

[12] Xiong, C., Rong, Y., Koganti, R.P., Zaluzec, M.J., Wang, N. (2002). Geometric variation prediction in automotive assembling, Assembly Automation, Vol. 22, No. 3, 260-269, doi: 10.1108/01445150210436473.

[13] Carlson, J.S., Söderberg, R. (2003). Assembly root cause analysis: A way to reduce dimensional variation in assembled products, International Journal of Flexible Manufacturing Systems, Vol. 15, No. 2, 113-150, doi: 10.1023/ A:1024453207632.

[14] Kim, I.S., Son, K.J., Yang, Y.S., Yaragada, P.K.D.V. (2003). Sensitivity analysis for process parameters in GMA welding processes using a factorial design method, International Journal of Machine Tools and Manufacture, Vol. 43, No. 8, 763-769, doi: 10.1016/S0890-6955(03)00054-3.

[15] Kazmer, D., Roser, C. (1999). Evaluation of product and process design robustness, Research in Engineering Design, Vol. 11, No. 1, 20-30, doi: 10.1007/s001630050002.

[16] Nejad, M.K., Vignat, F., Villeneuve, F. (2012). Tolerance analysis in machining using the model of manufactured part (MMP) - comparison and evaluation of three different approaches, International Journal of Computer Integrated Manufacturing, Vol. 25, No. 2, 136-149, doi: 10.1080/0951192X.2011.627943.

[17] King, J.P., Jewett, W.S. (2010). Robustness development and reliability growth: Value adding strategies for new products and processes, Prentice Hall, New Yersey, USA.

[18] Göhler, S.M., Eifler, T., Howard T.J. (2016). Robustness metrics: Consolidating the multiple approaches to quantify robustness, Journal of Mechanical Design, (In Press), doi: 10.1115/1.4034112.

[19] Augusto, O.B., Bennis, F., Caro, S. (2012). Multiobjective engineering design optimization problems: A sensitivity analysis approach, Pesquisa Operacional, Vol. 32, No. 3, 575-596, doi: 10.1590/S0101-74382012005000028.

[20] Hung, T.-C., Chan, K.-Y. (2013). Multi-objective design and tolerance allocation for single- and multi-level systems, Journal of Intelligent Manufacturing, Vol. 24, No. 3, 559-573, doi: 10.1007/s10845-011-0608-3.

[21] Bosire, J., Wang, S., Khasawneh, M., Gandhi, T., Srihari, K. (2016). Designing an integrated surgical care delivery system using axiomatic design and petri net modeling, Advances in Healthcare Informatics and Analytics, Vol. 19, 73-101, doi: 10.1007/978-3-319-23294-2 4.

[22] Ghanmi, S., Guedri, M., Bouazizi, M.-L., Bouhaddi, N. (2011). Robust multi-objective and multi-level optimization of complex mechanical structures, Mechanical Systems and Signal Processing, Vol. 25, No. 7, 2444-2461, doi: 10.1016/i.ymssp.2011.02.011.

[23] Tong, C., Graziani, F. (2008). A practical global sensitivity analysis methodology for multi-physics applications, Computational Methods in Transport: Verification and Validation, Vol. 62, 277-299, doi: 10.1007/978-3-54077362-7_12.

[24] Kackar, R.N. (1989). Off-line quality control, parameter design, and the Taguchi method. In: Dehnad, K. (ed.), Quality Control, Robust Design, and the Taguchi Method, Springer US, New York, USA, 51-76, doi: 10.1007/978-14684-1472-14

[25] Pedersen, S.N., Christensen, M.E., Howard, T.J. (2016). Robust design requirements specification: A quantitative method for requirements development using quality loss functions, Journal of Engineering Design, Vol. 27, No. 8, 544-567, doi: 10.1080/09544828.2016.1183163. 


\section{APEM}

Advances in Production Engineering \& Management Letnik 11 | Številka 3 | September 2016 | Strani 159-172 http://dx.doi.org/10.14743/apem2016.3.217
ISSN 1854-6250

Spletna stran: apem-journal.org Izvirni znanstveni članek

\section{Sistem spremljanja proizvodnje za razumevanje robustnosti izdelka}

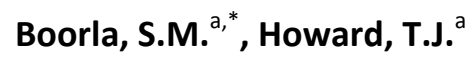

${ }^{a}$ Technical University of Denmark, Department of Mechanical Engineering, Denmark

\section{POVZETEK}

V proizvodnji zagotavljamo konstantno kakovost (lastnost) izdelka tako, da zagotovimo, da so lastnosti njegovih sestavin v okviru proizvodnih specifikacij. Lastnost izdelka se preverja po končni montaži. Vendar pa ta pristop ne nadzira robustnosti lastnosti izdelka, tj. koliko se bo razlikoval od specificiranih lastnosti. $V$ pričujoči raziskavi je predlagan model za napovedovanje lastnosti izdelka, ki upošteva zasnovo, montažo in procesne parametre neposredno iz proizvodnje. To omogoča, da v proizvodnji ohranjamo končne lastnosti izdelka namesto zgolj lastnosti njegovih sestavnih delov. Da bi prikazali kako je lahko sistem spremljanja uspešen pri vodenju korektivnih akcij za izboljšanje lastnosti izdelka smo uporabili PRECI-IN študijo primerov. Za podkrepitev teze, da lahko sistem spremljanja bistveno skrajša čas za identifikacijo, načrtovanje in izvedbo korektivnih akcij povezanih s kakovostjo izdelka, smo izvedli dve študiji primerov iz različnih industrijskih okolij. Intervjuji s strokovnjaki s področja kakovosti so pokazali, da je značilen čas, potreben za korektivne akcije, znašal v obeh primerih okoli sedem dni. Z uporabo sistema za spremljanje (PRECI-IN) pa so podobne korektivne akcije bile izvedene domala takoj.

\section{PODATKI O ČLANKU}

Ključne besede:

Robustnost izdelka

Spreminjanje lastnosti

Sistem za spremljanje robustnosti

Konsistentnost lastnosti

Robustnost element-element

*Kontaktna oseba:

srimbo@mek.dtu.dk

(Boorla, S.M.)

Zgodovina članka:

Prejet 27. maja 2016

Popravljen 5. avgusta 2016

Sprejet 17. avgusta 2016 